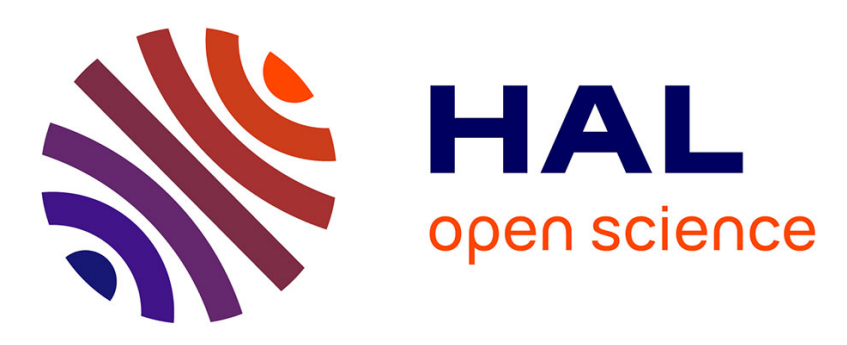

\title{
Poroelasticity of a micro-heterogeneous material saturated by two immiscible fluids
}

\author{
Siavash Ghabezloo, Sahar Hemmati
}

\section{To cite this version:}

Siavash Ghabezloo, Sahar Hemmati. Poroelasticity of a micro-heterogeneous material saturated by two immiscible fluids. International Journal of Rock Mechanics and Mining Sciences, 2011, 48 (8), pp.1376-1379. 10.1016/j.ijrmms.2011.10.003 . hal-00689726

\section{HAL Id: hal-00689726 \\ https://hal.science/hal-00689726}

Submitted on 19 Apr 2012

HAL is a multi-disciplinary open access archive for the deposit and dissemination of scientific research documents, whether they are published or not. The documents may come from teaching and research institutions in France or abroad, or from public or private research centers.
L'archive ouverte pluridisciplinaire HAL, est destinée au dépôt et à la diffusion de documents scientifiques de niveau recherche, publiés ou non, émanant des établissements d'enseignement et de recherche français ou étrangers, des laboratoires publics ou privés. 


\title{
Poroelasticity of a micro-heterogeneous material saturated by two immiscible fluids
}

\author{
Siavash Ghabezloo ${ }^{\mathrm{a}}$, Sahar Hemmati ${ }^{\mathrm{b}}$ \\ ${ }^{a}$ Université Paris-Est, Laboratoire Navier, CERMES, Ecole des Ponts ParisTech, Marne-la-Vallée, France \\ ${ }^{b}$ Laboratoire MSS-Mat, CNRS UMR 8579, Ecole Centrale Paris, France
}

\begin{abstract}
The anisotropic poroelasticity framework presented by Aichi and Tokunaga (Int. J. Rock Mech. Min. Sci. 48(4) (2011) 580-584) for a porous material saturated by two immiscible fluids is generalized for micro-heterogeneous materials by introducing one additional poroelastic parameter. This framework is based on Coussy's thermodynamic framework, but is re-written using material parameters that are easier to evaluate experimentally. The presented generalization permits using the constitutive relations for modelling the behaviour of geomaterials with a heterogeneous solid phase composed of several constituents. The constitutive relations are given also for special cases of isotropic porous material saturated by two immiscible fluids or by one fluid.
\end{abstract}

Keywords: Poroelasticity, two phase fluid, constitutive relations, unsaturated, anisotropy

\section{Introduction}

The modelling of the mechanical behaviour of unsaturated porous materials is important for various applications in geotechnical engineering, petroleum geomechanics, biomechanics, etc. Based on the thermodynamics theory and Lagrangian saturation concept, Coussy [1,2] presented a solid framework for modelling the elastoplastic behaviour of unsaturated porous media. This framework is presented based on material properties that are very difficult to evaluate experimentally. Aichi and Tokunga [3] represented this theoretical framework for a more general case of anisotropic poroelasticity using material properties that are easier to evaluate experimentally. By means of a series of thought experiments, these authors established relationships for the unsaturated poroelasticity material parameters used in Coussy's framework as functions of porosity, saturated poroelasticity material parameters (drained stiffness and Biot effective stress coefficient tensors), Bishop effective stress coefficient and degree of saturation. But these relationships are limited to micro-homogeneous porous materials for which the solid phase is made up of one single constituent. It is well-known that this is not the case for many geomaterials constituted by a heterogeneous solid phase. The aim of this work is to generalize the presented constitutive relations in [3] by taking into account the heterogeneity of the solid phase of the porous material. This is done by introducing an additional poroelastic parameter into the formulations.

\section{Poroelastic constitutive relations for unsaturated media}

The poroelastic constitutive relations presented in the following are written considering an infinitesimal representative element of porous material. The material with total volume $V$ is composed of a solid phase with volume $V_{s}$ and a connected pore volume with volume $V_{\phi}$. The occluded pore volume and the fluid filling it are considered to be a part of the solid phase. The solid phase itself may be composed of one or several constituents. The Lagrangian porosity is defined as $\phi=V_{\phi} / V_{0}$ and its variation is expressed by $\varphi=\phi-\phi_{0}$, where the subscript 0 is for referring to the initial

\footnotetext{
Email address: siavash.ghabezloo@enpc.fr (Siavash Ghabezloo)

${ }^{1}$ Corresponding author: Siavash Ghabezloo, Laboratoire Navier-CERMES, Ecole des Ponts ParisTech, 6-8 Av. Blaise Pascal, Cité Descartes, Champs sur Marne, 77455 Marne la Vallée Cedex 2, France
} 
state. It is assumed that the pore volume is composed of two parts, each one fully saturated by a fluid. These pore volumes are referred to in the following by subscripts $w F$ and $n w F$, respectively for wetting fluid and non-wetting fluid, so that $\phi=\phi_{w F}+\phi_{n w F}$. From this relation one obtains

$$
\varphi=\phi-\phi_{0}=\varphi_{w F}+\varphi_{n w F}
$$

The Lagrangian partial saturations for wetting and non-wetting fluids represent the fraction of the pore volume occupied by each fluid:

$$
S_{w F}=\frac{\phi_{w F}}{\phi_{0}} \quad ; \quad S_{n w F}=\frac{\phi_{n w F}}{\phi_{0}} \quad ; \quad S_{w F}+S_{n w F}=1
$$

The poroelastic constitutive relations for a general case of a fully anisotropic unsaturated material are given below $[1,2,3]:$

$$
\begin{gathered}
\sigma_{i j}=C_{i j k l} \varepsilon_{k l}-\alpha_{w F i j} p_{w F}-\alpha_{n w F i j} p_{n w F} \\
\varphi_{w F}=\alpha_{w F i j} \varepsilon_{i j}+\frac{1}{N_{w F-w F}} p_{w F}+\frac{1}{N_{w F-n w F}} p_{n w F} \\
\varphi_{n w F}=\alpha_{n w F i j} \varepsilon_{i j}+\frac{1}{N_{w F-n w F}} p_{w F}+\frac{1}{N_{n w F-n w F}} p_{n w F}
\end{gathered}
$$

where $C_{i j k l}$ is the stiffness tensor and $\alpha_{w F i j}, \alpha_{n w F i j}, N_{w F-w F}, N_{w F-n w F}, N_{n w F-n w F}$ are material constants that are equivalent to Biot effective stress coefficient tensor $\alpha_{i j}$ and Biot modulus $N$ in saturated conditions. As mentioned by Aichi and Tokunga [3], these material parameters defined for unsaturated poroelasticity are not directly relevant to those which can be measured by laboratory experiments. In other words, an experimental evaluation of these parameters is very difficult, if not impossible. To overcome this difficulty, Aichi and Tokunga [3] propose an interesting method, by means of a series of thought experiments, to establish relations among these unsaturated poroelasticity material properties with other parameters that are easier to evaluate experimentally. These relations which are presented in [3] for the case of a micro-homogeneous porous material, are generalized in the following section for the case of a micro-heterogeneous porous material for which the solid phase is composed of several constituents. The derivation of these relations is done using the same method as in [3] and for this reason the structure of the following section is chosen similar to section (3) of [3].

\section{Theoretical relationships among poroelastic coefficients}

\subsection{Uniform pore pressure build-up}

When the pressure increments in wetting and non-wetting fluid are the same, equations 3 to 5 must be reduced to the saturated poroelasticity equations, $\sigma_{i j}=C_{i j k l}^{\prime} \varepsilon_{k l}-\alpha_{i j} p$ and $\varphi=\alpha_{i j} \varepsilon_{i j}+p / N[1,3]$. In these equations $C_{i j k l}^{\prime}$ is the drained stiffness tensor, $\alpha_{i j}$ in Biot effective stress coefficient tensor and $N$ is Biot modulus. By assuming $p_{w F}=p_{n w F}=p$ in equation 3 and comparing with $\sigma_{i j}=C_{i j k l}^{\prime} \varepsilon_{k l}-\alpha_{i j} p$ one finds $C_{i j k l}=C_{i j k l}^{\prime}$ and $\alpha_{w F i j}+\alpha_{n w F i j}=\alpha_{i j}$. This latter equation permits writing

$$
\alpha_{w F i j}=\chi \alpha_{i j} \quad ; \quad \alpha_{n w F i j}=(1-\chi) \alpha_{i j}
$$

where $\chi$ is a constant depending on the Lagrangian saturation of wetting fluid. Replacing these relations in the constitutive relation 3 gives

$$
\sigma_{i j}=C_{i j k l}^{\prime} \varepsilon_{k l}-\alpha_{i j}\left[\chi p_{w F}+(1-\chi) p_{n w F}\right]
$$

which shows that the parameter $\chi$ is equivalent to Bishop [4] effective stress coefficient. Inserting equations 4 and 5 and $p_{w F}=p_{n w F}=p$ in equation 1 and comparing with the saturated poroelasticity equation $\varphi=\alpha_{i j} \varepsilon_{i j}+p / N$, results in the following relation between the unsaturated poroelasticity constants and Biot modulus $N$ for the saturated case [1]: 


$$
\frac{1}{N_{w F-w F}}+\frac{2}{N_{w F-n w F}}+\frac{1}{N_{n w F-n w F}}=\frac{1}{N}
$$

\subsection{Unjacketed condition}

The unjacketed condition is defined as $p_{w F}=p_{n w F}=p$ and $\sigma_{i j}=-p \delta_{i j}$. Under this condition, equal increments of confining pressure and pore pressure are simultaneously applied to the sample, as if the sample was submerged, without a jacket, into a fluid under the pressure $p$. Terzaghi effective stress $\sigma_{i j}^{\prime}=\sigma_{i j}+p \delta_{i j}$ in this condition remains constant. In the unjacketed condition a micro-homogeneous porous material would deform as if all the pores were filled with the solid component. The skeleton and the solid component experience a uniform volumetric strain $d V / V_{0}=$ $d V_{s} / V_{s 0}=d V_{\phi} / V_{\phi 0}$, so that we can write [3]:

$$
\varepsilon_{k k}=\frac{\varphi_{w F}}{\phi_{0} S_{w F}}=\frac{\varphi_{n w F}}{\phi_{0} S_{n w F}}
$$

When the solid phase is heterogeneous the skeleton and the solid component will experience different volumetric strains and the above relation is modified as following:

$$
\beta \varepsilon_{k k}=\frac{\varphi_{w F}}{\phi_{0} S_{w F}}=\frac{\varphi_{n w F}}{\phi_{0} S_{n w F}}
$$

where the parameter $\beta$ is a poroelastic parameter defined as the ratio of the volumetric strain of the pore volume to the one of the solid skeleton in unjacketed condition.

$$
\beta=\left(\frac{d V_{\phi} / V_{\phi 0}}{d V / V_{0}}\right)_{\substack{p_{w F}=p_{n w F}=p \\ \sigma_{i j}=-p \delta_{i j}}}
$$

In poroelasticity theory, two elastic moduli $K_{s}$ and $K_{\phi}$ are classically defined for unjacketed condition $[5,6,7]$.

$$
\frac{1}{K_{s}}=-\frac{1}{V_{0}}\left(\frac{\partial V}{\partial p}\right)_{\substack{p_{w F}=p_{n w F}=p \\ \sigma_{i j}=-p \delta_{i j}}} ; \quad \frac{1}{K_{\phi}}=-\frac{1}{V_{\phi 0}}\left(\frac{\partial V_{\phi}}{\partial p}\right)_{\substack{p_{w F}=p_{n w F}=p \\ \sigma_{i j}=-p \delta_{i j}}}
$$

The poroelastic parameter $\beta$ can thus be identified as:

$$
\beta=\frac{K_{s}}{K_{\phi}}
$$

The parameters $K_{s}$ and $K_{\phi}$ are equivalent respectively to $\kappa_{M}$ and $\kappa_{\phi}$ defined by Brown and Korringa [5] and $K_{s}^{\prime}$ and $K_{s}^{\prime \prime}$ defined by Rice and Cleary [6]. For a micro-homogeneous porous material $K_{s}=K_{\phi}=K_{m}$ where $K_{m}$ is the bulk modulus of the single solid constituent of the porous material. In the case of a porous material which is composed of two or more solids and therefore is heterogeneous at the micro-scale, the unjacketed modulus $K_{s}$ is some weighted average of the bulk moduli of solid constituents [7]. What this average should be is generally unknown, however, Ghabezloo and Sulem [8] evaluated the unjacketed modulus of Rothbach sandstone using Hill's [9] average formula and found a good accordance with the experimentally evaluated modulus. The modulus $K_{\phi}$ for such a material has a complicated dependence on the material properties. Generally it is not bounded by the elastic moduli of the solid components and can even have a negative sign if the bulk moduli of the individual solid components are greatly different one from another [10]. The modulus $K_{s}$ can be easily evaluated in an unjacketed test by measurement of the variations of the total volume of the tested sample. The variation of the pore volume of the sample in this test, evaluated from the quantity of fluids exchanged between the sample and the pore pressure generators could in principle give the modulus $K_{\phi}$. However experimental evaluation of this parameter is very difficult as the volume of the exchanged fluid has to be corrected for the effect of fluid compressibility, and also for the effect of the deformations of the pore pressure generator and drainage system in order to access to the variation of the pore volume of the sample $[11,12]$. However this parameter can be evaluated indirectly as a function of the other poroelastic parameters which can be measured independently, as presented in [11, 13]. It is also possible to evaluate this parameter using the homogenization method $[14,15]$. The parameter $\beta$ for a hardened cement paste is evaluated equal to 1.24 from the analysis of poromechanics tests [14] $\left(K_{s}=21.0 \mathrm{GPa}, K_{\phi}=16.9 \mathrm{GPa}\right)$ and equal to $1.1 \mathrm{using}$ the homogenization 
method [11]. From the experimental results of Hart and Wang [13] the parameter $\beta$ can be evaluated equal to 6.6 for Berea sandstone $\left(K_{s}=28.9 \mathrm{GPa}, K_{\phi}=4.4 \mathrm{GPa}\right)$ and 9.7 for Indiana limestone $\left(K_{s}=72.6 \mathrm{GPa}, K_{\phi}=7.5 \mathrm{GPa}\right)$. Based on the experimental results of Laurent et al. [16] cited in [13], the parameter $\beta$ can be evaluated between 0.33 and 0.56 for Vilhonneur limestone $\left(K_{s}=41\right.$ and $56 \mathrm{GPa}$ from two independent measurements, $K_{\phi}$ between 100 and $125 \mathrm{GPa})$.

By re-writing equations 3, 4 and 5 for the unjacketed condition $\left(p_{w F}=p_{n w F}=p\right.$ and $\left.\sigma_{i j}=-p \delta_{i j}\right)$ and using equations 10 and 6 the following relations are found between the Biot moduli for undsaturated condition and the other poroelastic parameters:

$$
\begin{gathered}
\frac{1}{N_{w F-w F}}+\frac{1}{N_{w F-n w F}}=\left(\alpha_{i j} \chi-\phi_{0} \beta S_{w F} \delta_{i j}\right) C_{i j k l}^{\prime}-1 \\
\frac{1}{N_{w F-w F}}+\frac{1}{N_{n w F-n w F}}=\left[\delta_{k l}-\alpha_{k l}\right)
\end{gathered}
$$

3.3. Build-up of the pressure of the wetting phase fluid under constant Lagrangian partial porosity of the non-wetting phase fluid and no deformation of the solid constituent

In this thought experiment the build-up of the pressure of the wetting phase fluid under constant Lagrangian partial porosity of the non-wetting phase fluid are considered as follows:

$$
p_{w F}=p ; \quad \varphi_{n w F}=0 ; \quad S_{w F}=\text { const. }
$$

Schematic representations of these conditions are presented in [3]. In addition to these conditions, the total stress is supposed to be:

$$
\sigma_{i j}=-\beta \phi_{0}\left[S_{w F} p+\left(1-S_{n w F}\right) p_{n w F}\right] \delta_{i j}
$$

By assuming $\beta=1$ the above equation is reduced to the one presented in [3]. Using equation (17), following the same way as presented in section 3.3 of [3], one can show that $\varphi_{w F}+\varphi_{n w F}=\varepsilon_{k k}$ and the strain of the solid constituent is zero. Finally, the following relations are obtained for the three Biot moduli (see [3] for more details on derivation):

$$
\begin{aligned}
& \frac{1}{N_{w F-n w F}}=\left(\alpha_{i j} \chi-\phi_{0} \beta S_{w F} \delta_{i j}\right) C_{i j k l}^{\prime-1}\left(\delta_{k l}-\alpha_{k l}\right) \\
& \times {\left[\alpha_{m n}(1-\chi)-\phi_{0} \beta\left(1-S_{w F}\right) \delta_{m n}\right]\left(\alpha_{m n}-\phi_{0} \beta \delta_{m n}\right)^{-1} } \\
& \frac{1}{N_{w F-w F}}=\left(\alpha_{i j} \chi-\phi_{0} \beta S_{w F} \delta_{i j}\right) C_{i j k l}^{\prime-1}\left(\delta_{k l}-\alpha_{k l}\right) \\
& \times\left(\alpha_{m n} \chi-\phi_{0} \beta S_{w F} \delta_{m n}\right)\left(\alpha_{m n}-\phi_{0} \beta \delta_{m n}\right)^{-1} \\
& \frac{1}{N_{n w F-n w F}}= {\left[\alpha_{i j}(1-\chi)-\phi_{0} \beta\left(1-S_{w F}\right) \delta_{i j}\right] C_{i j k l}^{\prime-1}\left(\delta_{k l}-\alpha_{k l}\right) } \\
& \times\left[\alpha_{m n}(1-\chi)-\phi_{0} \beta\left(1-S_{w F}\right) \delta_{m n}\right]\left(\alpha_{m n}-\phi_{0} \beta \delta_{m n}\right)^{-1}
\end{aligned}
$$

Equations 6, 18, 19 and 20 give relationships for unsaturated poroelasticity material parameters as functions of other parameters which are easier to evaluate experimentally. 


\section{Poroelastic constitutive relations for micro-heterogeneous materials}

\subsection{General case of anisotropic material}

Substituting equations $6,18,19$ and 20 in the constitutive relations 3,4 and 5 permit writing the poroelastic constitutive relations for an anisotropic micro-heterogeneous material:

$$
\begin{gathered}
\sigma_{i j}=C_{i j k l}^{\prime} \varepsilon_{k l}-\alpha_{i j}\left[\chi p_{w F}+(1-\chi) p_{n w F}\right] \\
\varphi_{w F}=\alpha_{i j} \chi \varepsilon_{i j}+\left(\alpha_{i j} \chi-\phi_{0} \beta S_{w F} \delta_{i j}\right) C_{i j k l}^{\prime}-1\left(\delta_{k l}-\alpha_{k l}\right)\left(\alpha_{m n} \chi-\phi_{0} \beta S_{w F} \delta_{m n}\right) \\
\times\left(\alpha_{m n}-\phi_{0} \beta \delta_{m n}\right)^{-1} p_{w F}+\left(\alpha_{i j} \chi-\phi_{0} \beta S_{w F} \delta_{i j}\right) C_{i j k l}^{\prime-1}\left(\delta_{k l}-\alpha_{k l}\right) \\
\times\left[\alpha_{m n}(1-\chi)-\phi_{0} \beta\left(1-S_{w F}\right) \delta_{m n}\right]\left(\alpha_{m n}-\phi_{0} \beta \delta_{m n}\right)^{-1} p_{n w F} \\
\varphi_{n w F}=\alpha_{i j}(1-\chi) \varepsilon_{i j}+\left(\alpha_{i j} \chi-\phi_{0} \beta S_{w F} \delta_{i j}\right) C_{i j k l}^{\prime-1}\left(\delta_{k l}-\alpha_{k l}\right) \\
\times\left[\alpha_{m n}(1-\chi)-\phi_{0} \beta\left(1-S_{w F}\right) \delta_{m n}\right]\left(\alpha_{m n}-\phi_{0} \beta \delta_{m n}\right)^{-1} p_{w F} \\
+\left(\alpha_{i j}(1-\chi)-\phi_{0} \beta\left(1-S_{w F}\right) \delta_{i j}\right) C_{i j k l}^{\prime-1}\left(\delta_{k l}-\alpha_{k l}\right) \\
\times\left[\alpha_{m n}(1-\chi)-\phi_{0} \beta\left(1-S_{w F}\right) \delta_{m n}\right]\left(\alpha_{m n}-\phi_{0} \beta \delta_{m n}\right)^{-1} p_{n w F}
\end{gathered}
$$

By assuming $\beta=1$ the above equations are reduced to the ones presented in [3].

\subsection{Special case of isotropy}

In the case of isotropy the constitutive relations are reduced to:

$$
\begin{aligned}
\sigma_{i j} & +\alpha\left[\chi p_{w F}+(1-\chi) p_{n w F}\right]=2 G \varepsilon_{i j}+\left(K-\frac{2 G}{3}\right) \varepsilon_{k k} \delta_{i j} \\
\varphi_{w F} & =\alpha \chi \varepsilon_{k k} \\
& +\frac{\left(\alpha \chi-\phi_{0} \beta S_{w F}\right)^{2}(1-\alpha)}{K\left(\alpha-\phi_{0} \beta\right)} p_{w F} \\
+ & \frac{\left(\alpha \chi-\phi_{0} \beta S_{w F}\right)\left[\alpha(1-\chi)-\phi_{0} \beta\left(1-S_{w F}\right)\right](1-\alpha)}{K\left(\alpha-\phi_{0} \beta\right)} p_{n w F} \\
\varphi_{n w F} & =\alpha(1-\chi) \varepsilon_{k k} \\
& +\frac{\left(\alpha \chi-\phi_{0} \beta S_{w F}\right)\left[\alpha(1-\chi)-\phi_{0} \beta\left(1-S_{w F}\right)\right](1-\alpha)}{K\left(\alpha-\phi_{0} \beta\right)} p_{w F} \\
& +\frac{\left[\alpha(1-\chi)-\phi_{0} \beta\left(1-S_{w F}\right)\right]^{2}(1-\alpha)}{K\left(\alpha-\phi_{0} \beta\right)} p_{n w F}
\end{aligned}
$$

\subsection{Special case of isotropy and saturation by one fluid}

For an isotropic porous material saturated by one fluid we have $\chi=1$ and $S_{w F}=1$. The constitutive relations in this case are reduced to:

$$
\begin{gathered}
\sigma_{i j}+\alpha p=2 G \varepsilon_{i j}+\left(K-\frac{2 G}{3}\right) \varepsilon_{k k} \delta_{i j} \\
\varphi=\alpha \varepsilon_{k k}+\frac{\left(\alpha-\phi_{0} \beta\right)(1-\alpha)}{K} p
\end{gathered}
$$


The term $\left(\alpha-\phi_{0} \beta\right)(1-\alpha) / K$ in equation (28) is equal to $1 / N$ where $N$ is Biot modulus. Replacing $\alpha=1-K / K_{s}$ and $\beta=K_{s} / K_{\phi}$ in this relation the expression of Biot modulus presented in $[17,14]$ is retrieved:

$$
\frac{1}{N}=\frac{\alpha}{K_{s}}-\frac{\phi_{0}}{K_{\phi}}
$$

In the case of a micro-homogeneous porous material composed of one single solid constituent we have $K_{s}=K_{\phi}$ and equation 29 is reduced to the well-known relation $1 / N=\left(\alpha-\phi_{0}\right) / K_{s}[17,18]$.

\section{Conclusions}

The anisotropic poroelasticity framework presented by Aichi and Tokunaga [3] for a porous material saturated by two immiscible fluids is generalized by taking into account the heterogeneity of the solid phase of the material. This is done by introducing one additional poroelastic parameter $\beta$ into the formulations. The parameter $\beta$ is defined as the ratio of the volumetric strain of the pore volume to the one of the solid skeleton in unjacketed condition. The assumption of a homogeneous solid phase is introduced in [3] and also in [1] to obtain the relationships between Biot modulus $N$ and the other poroelastic parameters. The presented generalization permits using the unsaturated poroelasticity constitutive relations for modelling the behaviour of anisotropic geomaterials with a heterogeneous solid phase. The constitutive relations are presented also for special cases of isotropic porous material saturated by two immiscible fluids as well as isotropic porous material saturated by one fluid.

\section{References}

[1] O. Coussy, Revisiting the constitutive equations of unsaturated porous solids using a lagrangian saturation concept, International Journal for Numerical and Analytical Methods in Geomechanics 31 (15) (2007) 1675-1694.

[2] O. Coussy, Mechanics and physics of porous solids, John Wiley \& Sons Inc, 2010.

[3] M. Aichi, T. Tokunaga, Thermodynamically consistent anisotropic constitutive relations for a poroelastic material saturated by two immiscible fluids, International Journal of Rock Mechanics and Mining Sciences 48 (4) (2011) 580 - 584.

[4] A. W. Bishop, The principle of effective stress, Teknisk Ukeblad 106 (1959) 859-863.

[5] R. Brown, J. Korringa, On the dependence of the elastic properties of a porous rock on the compressibility of the pore fluid, Geophysics 40 (1975) $608-616$.

[6] J. Rice, M. Cleary, Some basic stress diffusion solutions for fluid-saturated elastic porous media with compressible constituents, Rev. Geophys. Space Phys 14 (2) (1976) 227-241.

[7] J. Berryman, Effective stress for transport properties of inhomogeneous porous rock, J. Geophys. Res 97 (1992) $17409-17424$.

[8] S. Ghabezloo, J. Sulem, Stress dependent thermal pressurization of a fluid-saturated rock, Rock Mechanics and Rock Engineering 42 (2009) $1-24$.

[9] R. Hill, The Elastic Behaviour of a Crystalline Aggregate, Proceedings of the Physical Society A 65 (1952) 349-354.

[10] P. Berge, J. Berryman, Realizability of negative pore compressibility in poroelastic composites, Journal of applied mechanics 62 (4) (1995) 1053-1062.

[11] S. Ghabezloo, J. Sulem, S. Guédon, F. Martineau, J. Saint-Marc, Poromechanical behaviour of hardened cement paste under isotropic loading, Cement and Concrete Research 38 (12) (2008) 1424 - 1437.

[12] S. Ghabezloo, J. Sulem, Effect of the volume of the drainage system on the measurement of undrained thermo-poro-elastic parameters, International Journal of Rock Mechanics and Mining Sciences 47 (1) (2010) 60 - 68.

[13] D. J. Hart, H. F. Wang, Variation of unjacketed pore compressibility using gassmann's equation and an overdetermined set of volumetric poroelastic measurements, Geophysics 75 (1) (2010) N9-N18.

[14] S. Ghabezloo, Association of macroscopic laboratory testing and micromechanics modelling for the evaluation of the poroelastic parameters of a hardened cement paste, Cement and Concrete Research 40 (8) (2010) 1197 - 1210.

[15] S. Ghabezloo, Micromechanics analysis of thermal expansion and thermal pressurization of a hardened cement paste, Cement and Concrete Research 41 (5) (2011) $520-532$.

[16] J. Laurent, M. J. Bouteca, J. -P. Sarda, D. Bary, Pore-Pressure Influence in the Poroelastic Behavior of Rocks: Experimental Studies and Results, SPE Formation Evaluation 8 (2) (1993) 117 - 122.

[17] J. G. Berryman, G. W. Milton, Exact results for generalized gassmann's equations in composite porous media with two constituents, Geophysics 56 (12) (1991) 1950-1960.

[18] O. Coussy, Poromechanics, John Wiley \& Sons Inc, 2004. 